Boundary-Layer Meteorology manuscript No.

(will be inserted by the editor)

\title{
Modelling street-scale flow and dispersion in realistic winds - towards coupling with mesoscale meteorological models
}

\author{
Zheng-Tong Xie
}

Received: date / Accepted: date

\begin{abstract}
Further to our previous work - simulations of flow and dispersion in an oblique wind over the DAPPLE site (Xie ZT \& Castro IP, Atmospheric Environment, 2009, Vol.43, 2174-2185)- large-eddy simulations of flows and dispersion over the same site in a wind perpendicular to Marylebone Road and the windward surfaces of most of the buildings were performed. The DAPPLE site is located at the intersection of Marylebone Road and Gloucester Place in central London. In order to investigate the effects of wind direction on flows and dispersion, the velocity and scalar fields in the perpendicular wind were compared with those in the oblique wind. Furthermore, realistic wind conditions measured on the BT Tower at 190 $\mathrm{m}$ above street level were processed and used to drive the numerical simulations of flows and dispersion at the DAPPLE site. This leads to significant predictive improvements of the dispersion compared with field measurements, which provides validation and confidence for coupling mesoscale meteorological models, e.g. the UK Met Office's Unified Model and the NCAR's Weather Research \& Forecasting Model, with the street-scale large-eddy simulation of urban environments.
\end{abstract}

Keywords Large-eddy simulation · Neighbourhood scale · Urban dispersion · Wind variation

\section{Introduction}

It is known that wind-direction effects on flows over a single cube and a group of staggered cubes are significant (Lim et al., 2007; Davidson et al., 1995; Claus et al., 2009). Using large-eddy simulations, Claus et al. (2009) modelled the wind-direction effects on flows over a group of staggered cubes, and found that the mean flow within the lower part of the canopy showed only a weak dependence on the direction of the wind aloft, but the variations of the surface drag and of the parameters of the logarithmic wind law

Zheng-Tong Xie

School of Engineering Sciences

University of Southampton, SO17 1BJ, UK

Tel: +44(0)23 80594493

Fax: +44(0)2380593058

E-mail: z.xie@soton.ac.uk 
were non-linear and non-monotonic with the variation of wind angle from $0^{\circ}$ to $90^{\circ}$. This highlights the importance of taking the wind direction into account when studying urban flows.

Wind-direction effects on dispersion over a group of obstacles are not negligible either. Belcher (2005) commented that the differences in dispersion over an array of long cuboids (i.e. an aspect ratio $W / H=4$ of width and height) under $90^{\circ}$ and $60^{\circ}$ winds were 'striking'. The plume width grew more rapidly in the oblique wind than in the perpendicular wind (MacDonald et al., 1998; Belcher, 2005), because of stronger near-field dispersion and topological dispersion enhancing the far-field dispersion. Belcher (2005) suggested that the wind-direction fluctuations increased the potency of topological dispersion, particularly for streets with a large aspect ratio $W / H$, e.g. greater than 4. Davidson et al. (1995) conducted a set of field experiments of a neutrally buoyant plume released upwind of an array of cubes, and observed that the lateral fluctuations in wind direction caused by large-scale eddies advected the centre of the plume from one obstacle to another. A number of secondary sources (sometimes known as wake sources behind the obstacles) co-existed within the array as the plume meandered in the upstream wind. Certainly, the large-time scale fluctuations of wind direction strengthened the effects of the secondary sources. Using direct numerical simulations, Branford et al. (2011) recently investigated the dispersion of a point-source release in a regular array of cubes in three different pressure gradient directions (i.e. $0^{\circ}, 30^{\circ}$ and $45^{\circ}$ ). This largely helped to further understand the processes affecting the plume structure, e.g. channelling, lateral dispersion, detrainment, secondary-source dispersion and plume skewing.

It is also known that the urban boundary layer, the rural boundary layer, the city scale flow and the regional weather are coupled aerodynamically and thermodynamically (Britter and Hanna, 2003; Fernando et al., 2010). This again suggests that it is of great importance to consider the wind-direction effects on urban flows and dispersion. It is known that the mean concentration obtained from small-scale physical or numerical models can be much greater than that obtained in full- scale field experiments (Cheng and Robins, 2004). The discrepancy might be due to the variation of wind direction and magnitude, thermal buoyancy effects or the myriad small roughness elements in the full-scale urban region (Xie and Castro, 2010). Whilst it might be straightforward to consider simulating the street scale and the mesoscale motions together (Mochida et al., 2010; Yamada, 2010), two questions must first be answered.

The first question is: what is the most appropriate approach for modelling street-scale flows and dispersion. Given the strong gradients caused by the presence of buildings and street scales, a resolution down to one metre is required (Xie and Castro, 2006; Fernando et al., 2010). Direct numerical simulation (DNS) is the most accurate computational fluid dynamics (CFD) approach (Coceal et al., 2006), but it requires enormous computer resources for high Reynolds number $(R e)$ flows and is not suitable for urban flows in full scale. Large-eddy simulations (LES) with wall modelling are less resource demanding than DNS. Generally speaking, LES is more accurate than the statistical models (RANS) for flows over groups of sharp edged obstacles, e.g. in the prediction of separation bubbles (Niceno and Hanjalić, 2002; Tseng et al., 2006; Xie and Castro, 2006; Santiago et al., 2010). In contrast to RANS methods, LES results of three groups (Hellstena and Rautaheimo, 1999) all showed satisfactory agreement with measurements. More recently, a number of LES calculations, e.g. Hanna et al. (2002); Stoesser et al. (2003); Kanda et al. (2004), as well as our own, have been performed for flows over groups of cubes, demonstrating their superiority for such applications. There is thus convincing evidence that LES is the most promising tool for computing unsteady, three-dimensional flows at high $R e$, and it is used in the present work. Indeed, LES with a proper wall treatment is arguably the only approach likely to produce reliable numerical data over a range of problems and yet be within the affordability of the current and near-future computer power for genuine urban geometries (Tseng et al., 2006; Xie and Castro, 2009). 
The second question relates to the coupling of street-scale and mesoscale motions. The traditional approach usually adopted in mesoscale models consists in modifying the roughness length and the thermal properties of the ground, but the latter in particular are not easy to obtain. Another disadvantage of this approach, particularly in urban situations, is that this cannot in general predict accurately the vertical structure of the turbulent momentum and heat fluxes. The main reason is that in this approach the only sink of momentum and sink/source of heat are placed at the ground and are not distributed up to the building height. To improve this, Martilli (2002) and Solazzo et al. (2010) used a simple urban model based on a parametrized formulation of the sink of momentum and sink/source of heat at several levels within the urban canopy, to couple with a mesoscale meteorological model. Such an improved parametrization method is not able to predict dispersion at street scales. Inevitably CFD approaches should be used for such purposes.

It is a great challenge to predict flows and dispersion in full- scale urban environments, e.g. from mesoscale to street-scale (down to $1 \mathrm{~m}$ ). Historically the mesoscale meteorological model and the CFD codes (e.g. for street-scale motions) were developed separately. Their objectives and approaches were significantly different (Yamada, 2010). In particular for urban environment applications, the order of scales varies from one kilometre to one metre. Indeed it is surely too ambitious to simulate all of these scales simultaneously in the presence of buildings. One of the alternatives is to up-scale CFD models to include the influences of mesoscale motions. So it is of great interest to numerically predict urban flows and dispersion within a genuine city area driven by realistic weather conditions. However, there are a few more open questions to be answered. For example, there is a big gap between the minimum time scale of a mesoscale meteorological model and the integral time scale of turbulence in a CFD model, in particular large-eddy simulations.

This paper focuses on studying the effect on dispersion of variations of wind direction having frequencies lower than those of the dominant turbulent eddies in the approaching boundary- layer flow. We aim eventually to use the mesoscale meteorological model data to provide boundary conditions to drive the LES with a resolution down to one metre, whereas the horizontal spatial resolution of the former is on the order of one kilometre. This three-order of magnitude range in scale is much larger than can sensibly be approached using nesting techniques. (That used by Moeng et al., 2007, for example, has a grid ratio of only three.) This requires a sophisticated technique, i.e. turbulence inflow generation, to bridge the gap of grid resolution.

Inflow generators (e.g. Xie and Castro, 2008) require detailed information of the scales greater than the turbulence integral scales. However, again there is a significant lack of information for time scales between the smallest scale of the mesoscale meteorological model and the dominant turbulence scale. The unresolved issue is how to fill the gap to provide inflow information for the LES. As a start, rather than using the mesoscale meteorological model data, the DAPPLE wind data (Wood et al., 2009) with high time resolution on the BT Tower were post-processed and used to drive LES computations. It is this methodology, and the results, which are reported herein.

In the current study, the recently developed inflow technique (Xie and Castro, 2008) was applied to large-eddy simulation of flow and dispersion over the DAPPLE field site located at the intersection of Marylebone Rd and Gloucester Pl in Central London (Arnold et al., 2004). We chose the DAPPLE site as the test case because, (1) there are large quantities of field and wind-tunnel data available for validation; (2) the comprehensive genuine urban geometry was composed of staggered, aligned and square arrays of blocks with non-uniform height and non-uniform base, street canyons and intersections; (3) the DAPPLE site, with a linear scale of approximately one kilometre, was within the neighbourhood scale. The neighbourhood scale (i.e. 1000 - $2000 \mathrm{~m}$ ) is a spatial scale that one may consider comprises a comprehensive urban geometry, a scale at which a gross parametrization of the flow for mesoscale models can be at- 
tempted, and a scale at which detailed computational and wind-tunnel studies are feasible. It is also a scale over which a degree of statistical homogeneity may be anticipated; the city then is seen as being composed of a collection of these neighbourhoods. It is to be noted that the domain size $(1000 \mathrm{~m})$ is approximately the horizontal grid size of operational mesoscale meteorological models. This suggests that the approaches developed here can be easily transplanted for the one-way coupling between the mesoscale meteorological model and the street-scale LES, provided statistical information on the scales greater than the turbulence integral scale is available.

In our previous work, LES was applied to study flows and dispersion over the DAPPLE site for the prevailing wind direction of $-51^{\circ}$ bearing clockwise to the Marylebone $\mathrm{Rd}$ direction, approximately a south-west wind. In order to investigate the effect of wind direction, dispersion in a $-90^{\circ}$ wind, approximately a southerly wind, was investigated. Then realistic wind conditions measured at the BT Tower were used to drive the LES computations. In the following, Section 2 briefly presents the governing equations of the LES and Section 3 presents numerical details, including geometry, mesh and boundary conditions. Section 4 presents the comparison of flows and dispersion for the $-90^{\circ}$ and $-51^{\circ}$ winds, and Section 5 presents results obtained by using BT Tower data. Conclusions and final remarks are presented in Section 6.

\section{Governing Equations of Flows and Scalar Dispersion}

Large-eddy simulation (LES) resolves only the large-scale fluid motions and models the subgrid-scale (SGS) motions through filtering the Navier-Stokes equations. The SGS eddies provide the high frequency content at the upper end of the spectrum and thus, provided the grid is fine enough, contribute very little to the total turbulent kinetic energy. A brief description of the governing equations is given here, while more details can be found in Xie and Castro (2006), hereafter denoted by XC.

The filtered continuity and Navier-Stokes equations are written as follows,

$$
\begin{gathered}
\frac{\partial \bar{u}_{i}}{\partial x_{i}}=0 \\
\frac{\partial \bar{u}_{i}}{\partial t}+\frac{\partial \bar{u}_{i} \bar{u}_{j}}{\partial x_{j}}=-\frac{1}{\rho}\left(\frac{\partial \bar{p}}{\partial x_{i}}\right)+\frac{\partial}{\partial x_{j}}\left(\frac{\tau_{i j}}{\rho}+v \frac{\partial \bar{u}_{i}}{\partial x_{j}}\right) .
\end{gathered}
$$

The dynamical quantities, $\bar{u}_{i}, \bar{p}$ are the resolved-scale (filtered) velocity and pressure respectively, $\rho$ is the fluid density. $v$ is the kinematic molecular viscosity and $\tau_{i j}$ is the subgrid-scale (SGS) Reynolds stress. The Smagorinsky SGS model was used with $C_{s}=0.1$,

$$
\tau_{i j}-\delta_{i j} \tau_{k k} / 3=2 \rho\left(C_{s} \Delta\right)^{2}\left(2 \bar{s}_{m n} \bar{s}_{m n}\right)^{1 / 2} \bar{s}_{i j},
$$

where $\bar{s}_{i j}=\frac{1}{2}\left(\frac{\partial \bar{u}_{i}}{\partial x_{j}}+\frac{\partial \bar{u}_{j}}{\partial x_{i}}\right), \Delta$ is taken as the cubic root of the cell volume, $\tau_{k k}$ is modelled according to a closure similar to the one devised by Yoshizawa (1986), and $\delta_{i j}$ is the Kronecker-delta. In the near-wall region, the Lilly damping function was also applied.

For cases where the fine eddies in the vicinity of the wall are important, it is recommended that $\mathscr{N}_{1}^{+}$ is $\sim 1\left(\mathscr{N}_{1}^{+}\right.$is the distance in wall units between the centroid of the first cell and the wall assuming the 

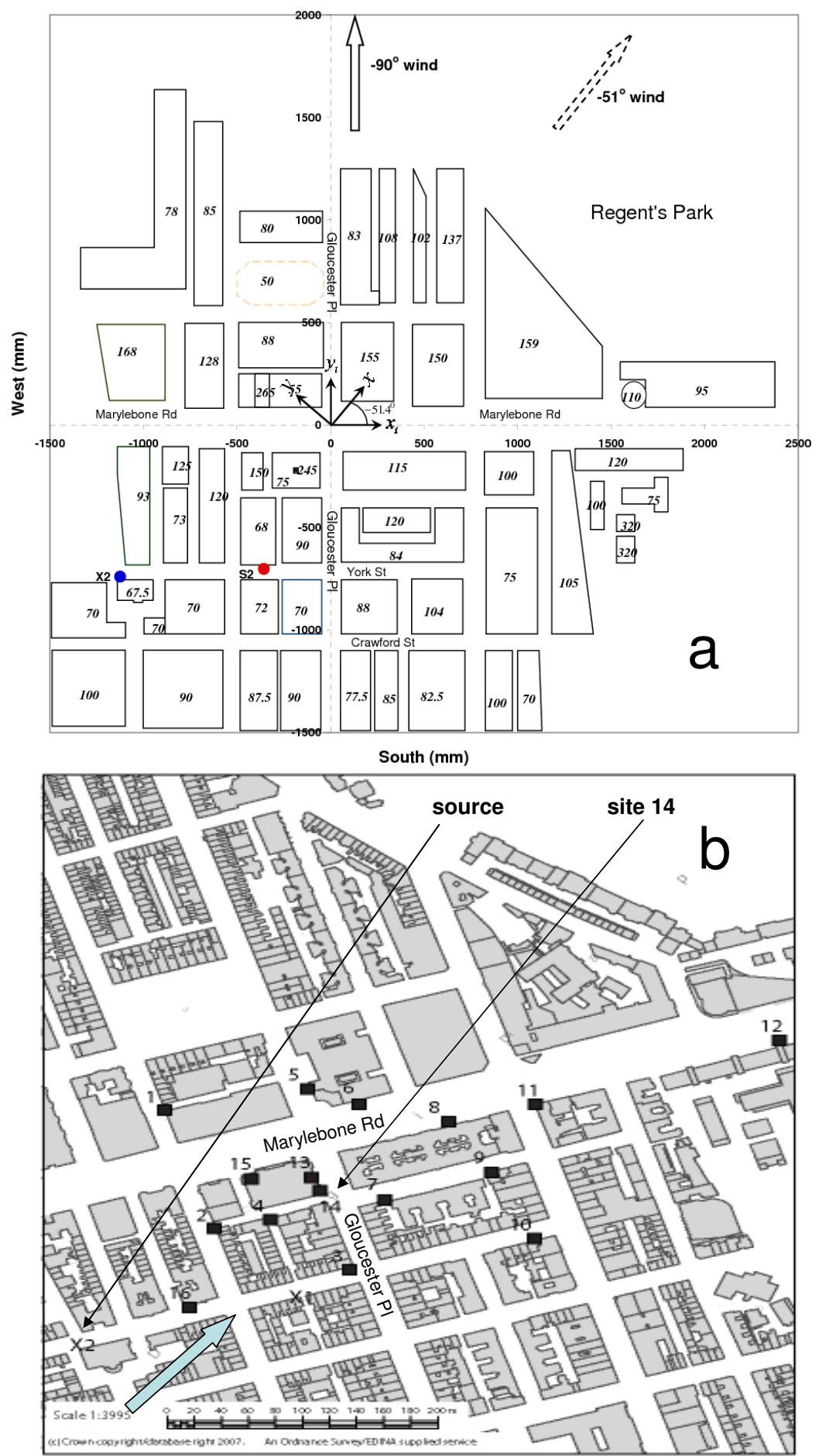

Fig. 1 Plan view of the DAPPLE site. (a) 1:200 wind-tunnel model (WTM): numbers in italics on each building block indicate its height in $\mathrm{mm}$; the model coordinates are marked in $\mathrm{mm}$, with $x_{t}$ from west to east, $y_{t}$ from south to north and $z$ from ground to top respectively; $x, y, z$ are the computational coordinates (Fig. 2). (b) Full-scale field experiment site: $X 1, X 2$, source sites; square symbols, sampling sites. 


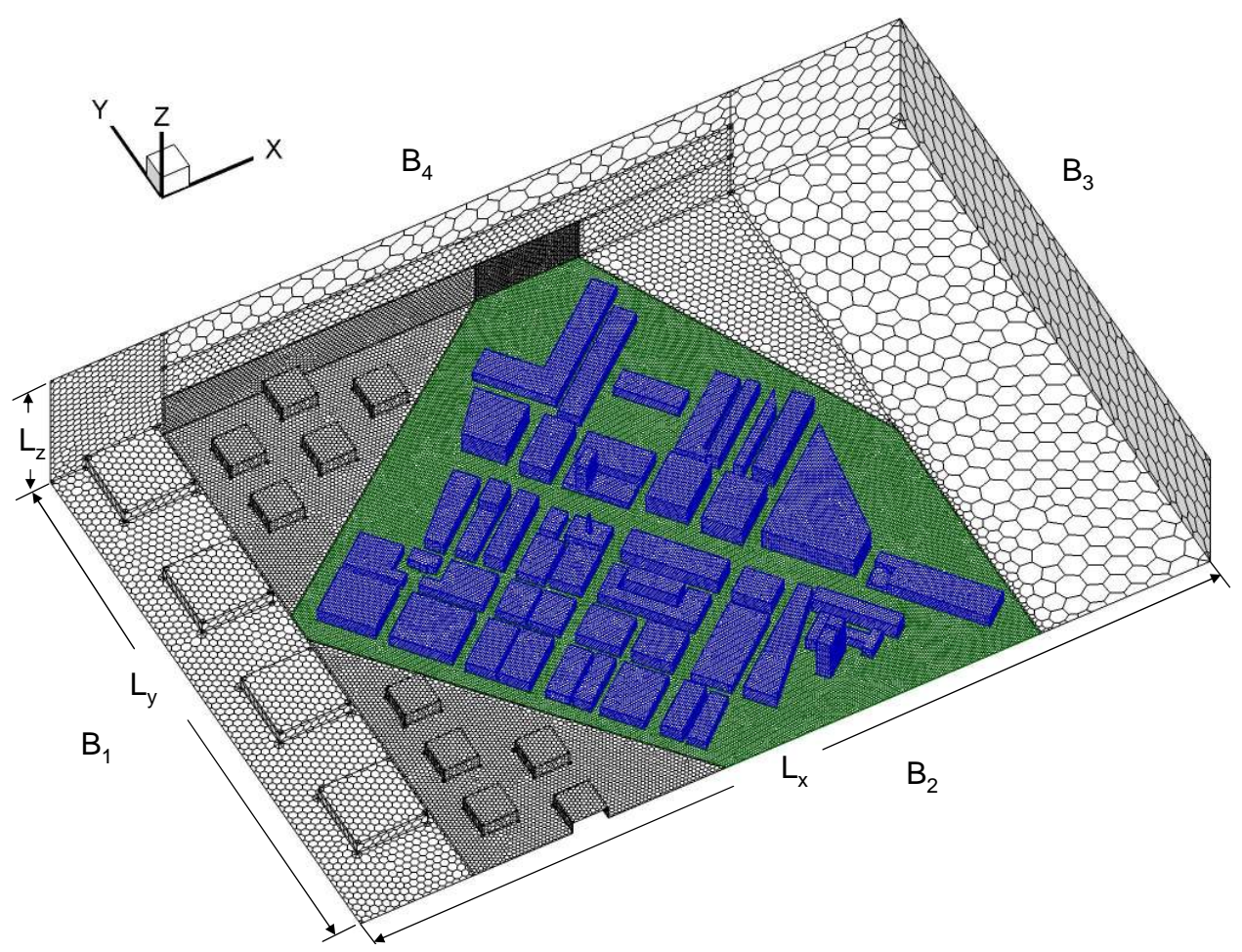

Fig. 2 Computational domain with polyhedral mesh. The domain size $L_{x}=6 \mathrm{~m}, L_{y}=4 \mathrm{~m}, L_{z}=1 \mathrm{~m}$ in WTM scale.

$\mathscr{N}$ coordinate is normal to the wall). Note, however, that for a complex geometry, where separation and attachment processes occur, it is impossible to satisfy this criterion everywhere. We argue that, unlike the situation for smooth-wall flows, it is in fact not necessary, at least for obtaining overall surface drag and the turbulent motions at the scale of the roughness elements (buildings), which turn out to be dominant. We have shown earlier that for this type of flow the precise surface condition is unimportant for capturing the element-scale flows and surface drag (see XC).

The filtered scalar transport equation is written as follows,

$$
\frac{\partial \bar{c}}{\partial t}+\frac{\partial \bar{u}_{j} \bar{c}}{\partial x_{j}}=\frac{\partial}{\partial x_{j}}\left(q_{j}+K_{m} \frac{\partial \bar{c}}{\partial x_{j}}\right)+S,
$$

where $\bar{c}$ is resolved-scale (filtered) scalar, $K_{m}$ is the molecular diffusivity, $q_{j}=K_{s} \frac{\partial \bar{c}}{\partial x_{j}}$ is the subgridscale (SGS) scalar flux, where $K_{s}$ is the subgrid turbulent diffusivity, $S$ is the source term, a function of space and time. So far most studies for concentration dispersion problems have applied a subgrid eddy viscosity combined with a subgrid-scale Schmidt number, which are set as constant or calculated dynamically. In the present study, we adopt this approach using a constant subgrid-scale Schmidt number of unity,

$$
K_{s}=v_{s} / S c_{s}
$$

where $v_{S}$ is the subgrid viscosity calculated in Eq. 2, and $S c_{s}$ is the subgrid Schmidt number. 


\section{Numerical Settings}

The entire LES model was implemented as described in XC. Crucially, the discretization for all terms in Eqs. 1 and 3 was second-order accurate in both space and time. To solve these equations, a second-order backward implicit scheme in time, a second-order central difference scheme for the diffusion terms in space, and a second-order Monotone Advection and Reconstruction Scheme (MARS) (STAR-CD, 2009) for the convective terms in space were applied. The MARS scheme is used to capture the sharp gradients at the edge of the scalar plume and to avoid generating spurious negative concentrations.

A description of the DAPPLE field experiments can be found in Arnold et al. (2004) and Wood et al. (2009), and a description of the wind-tunnel experiments can be found in Cheng and Robins (2004) and Carpentieri et al. (2009). We used LES to simulate flows and dispersion over the wind-tunnel model (WTM) - the 1:200 low resolution model, of which the plan view is shown in Fig. 1a. The model coordinates are marked in mm, with $x_{t}$ from west to east, $y_{t}$ from south to north and $z$ from ground to top respectively; $x$, $y, z$ are the computational coordinates (Fig. 2). The elliptic body with $50 \mathrm{~mm}$ height approximately at $x_{t}=$ $-250 \mathrm{~mm}, y_{t}=700 \mathrm{~mm}$ is a small park with trees. In the current paper, study of the effect of trees is not attempted. Since the small park is downwind of a tall building block and is two blocks downwind from the major intersection of Marylebone Rd with Gloucester Pl, its effect is unlikely to be significant to the flow and dispersion at the major intersection and in the region upwind. The arrows with solid and dashed line indicate the $-90^{\circ}$ wind and $-51^{\circ}$ wind respectively. Fig. $1 \mathrm{~b}$ shows the full-scale field experimental site.

Fig. 2 shows the computational domain with the coordinate origin at the ground at the Marylebone $\mathrm{Rd}$ - Gloucester Pl intersection, hereafter denoted the major intersection. The $x$ axis is approximately from south-west to north-east, while the $y$ axis is from south-east to north-west. The domain size is $L_{x}=6 \mathrm{~m}, L_{y}$ $=4 \mathrm{~m}, L_{z}=1 \mathrm{~m}$, which correspond to $1200 \mathrm{~m}, 800 \mathrm{~m}$ and $200 \mathrm{~m}$ respectively in full scale. Note the domain height is approximately nine times the mean building height $h_{m}$, which is similar to that of a simpler test case with random height blocks (Xie et al., 2008). The central part of the domain is the DAPPLE site. Upwind of the DAPPLE site fourteen artificial building blocks with a height $h_{m}$ were placed since the upwind urban geometry data was not available. It was found that the results within the DAPPLE site were not significantly sensitive to the arrangement of the artificial buildings (Xie and Castro, 2009). London's Regent's Park is downwind of the DAPPLE site and no artificial building blocks were installed in the outlet region.

Fig. 2 also shows the 3-D geometry of the building blocks (i.e. the blue ones) of the DAPPLE windtunnel model (WTM) with a mean height $h_{m}=110 \mathrm{~mm}$ and a packing density 0.5 , which is the area fraction occupied by buildings. Except for a few tall buildings, one small tower and one dome, most of the buildings are essentially of cuboid shape with various low heights. Their arrangement is mainly in staggered and aligned patterns with intersections and $\mathrm{T}$ junctions. A street canyon pattern is also evident and is more dominant for south-north streets than for east-west streets. Also the surface mesh of the building blocks is presented to indicate the resolution down to $h_{m} / 20,1 \mathrm{~m}$ in full scale, which is almost uniform (except the first grid from the solid wall) in the DAPPLE site up to $z / h_{m}=3$.

It is to be noted that polyhedral meshes are more flexible than hexahedral meshes for such a complex geometry and more accurate and efficient than tetrahedral meshes for LES (Peric, 2004). So a polyhedral mesh with four grid sizes was used for the whole domain, identical to the medium mesh in Xie and Castro (2009), in which a numerical experiment on the sensitivity to grid resolution was conducted that showed that the medium mesh produced results in good agreement with those of a finer mesh. Again, an almost uniform grid was used in the DAPPLE site up to $z / h_{m}=3$ in order to maintain great accuracy in the region of most interest. 
The $-51^{\circ}$ wind in the domain was in the direction of the vector $(x, y, z) / R=(1,0,0)$, while the $-90^{\circ}$ wind was in the direction of the vector $(x, y, z) / R=\left(\sin 51^{\circ}, \cos 51^{\circ}, 0\right)$, where $R=\sqrt{x^{2}+y^{2}+z^{2}}$. (It is to be noted that wind direction is defined by bearing clockwise from the west-east Marylebone Rd direction.) Boundary $B_{1}$ in the $-51^{\circ}$ wind case and boundaries $B_{1}, B_{2}$ and $B_{4}$ in the $-90^{\circ}$ wind case were set as inlet, where an inflow approach (Xie and Castro, 2008) was used to generate turbulence fluctuations correlated in space and in time. Note that 'inlet boundary' simply means 'velocity specified at the boundary' but not necessarily 'velocity directed into the domain'. For example, at the boundary B4 the mean flow was outward for the $-90^{\circ}$ wind. In the $-51^{\circ}$ wind case, boundaries $B_{2}$ and $B_{4}$ were set with symmetric conditions. Boundary $B_{3}$ was set as outlet for the two cases. The upper boundary of the domain was set as a stressfree rigid wall and the other boundaries were all solid walls. In order to check any effects of the settings of boundary conditions, a numerical experiment for the $-90^{\circ}$ wind was performed with $B_{4}$ and $B_{3}$ set as outflow and inlet boundaries respectively. No significant difference of the results was observed. For the varying wind, the same boundary conditions as those for the $-90^{\circ}$ wind were used. Such boundary conditions were convenient for using realistic wind conditions to drive the LES when the wind direction varies (and also convenient for the coupling of the LES and mesoscale meteorological models).

The mean velocity, Reynolds stresses and the turbulence integral length scales used to generate inflow boundary conditions are the same as in Xie and Castro (2009). A detailed description of this approach for the DAPPLE site can be found in Xie and Castro $(2008,2009)$. In the wind tunnel, a number of twodimensional roughness elements of a height $20 \mathrm{~mm}$ were installed upstream of the DAPPLE site up to the wind-tunnel inlet. Vertical profiles of Reynolds stresses at various stations upstream of the DAPPLE site were measured, and no significant difference at different horizontal locations above $0.5 h_{m}$ was observed (Cheng and Robins, 2004; Carpentieri et al., 2009). We argue in Xie and Castro (2009) that the details of the Reynolds stresses specified at the inlet within the canopy are NOT important for LES to predict dispersion at the DAPPLE site, because of the feature of sharp edges of the buildings. Both viscous effects and resolution play a role in principle in the near wall regions. However, again these effects are not crucial for the prediction of dispersion in the DAPPLE site. By fitting the measured data, simple profiles of the mean velocity $U_{m}$ and the Reynolds stresses $\overline{u^{\prime} u^{\prime}}, \overline{w^{\prime} w^{\prime}}$ and $\overline{u^{\prime} w^{\prime}}$ at the inlet were obtained. Because of the lack of experimental data on $\overline{v^{\prime} v^{\prime}}$, the profile $\overline{v^{\prime} v^{\prime}}$ was estimated using the similarity between $\overline{v^{\prime} v^{\prime}}$ and the other Reynolds normal stresses of flows over rough surfaces (Xie and Castro, 2006).

We define $U_{r e f}$ as the freestream velocity for the wind-tunnel and LES models, and the mean velocity magnitude at the BT Tower height for the field case; $l_{x}, l_{y}$ and $l_{z}$ are the integral length scales in the streamwise, lateral and vertical directions respectively. The integral length scales were estimated following the wind-tunnel measurements for flows over a group of cubes (Castro et al., 2006). We generated the turbulence fluctuations in terms of components in a local coordinate system aligned with the wind direction and then projected the velocities on the computational domain coordinate for the inlet boundaries, e.g. $B_{1}$, $B_{2}$ and $B_{4}$. The Reynolds number $U_{r e f} h_{m} / v$ based on the freestream $U_{r e f}$ velocity, which was the velocity at height $L_{z}=1 \mathrm{~m}$, and the mean building height $h_{m}$ of the wind-tunnel model was approximately 18,000, which also the case for the LES model. All the numerical simulations were performed at the wind-tunnel model (WTM) scale. The timestep $\Delta t$ was initially set to $0.003125 \mathrm{~s}$, giving an averaged CFL number $\left(U_{r e f} \Delta t / \Delta x\right.$, where $\Delta x$ grid size) less than 0.5 . Results were in good agreement with those using a timestep $0.00625 \mathrm{~s}$, which was therefore used for most of the computations. For the $-51^{\circ}$ and $-90^{\circ}$ wind directions, the initial duration of most of the large-eddy simulations was $62 \mathrm{~s}$ with subsequent averaging over at least $62 \mathrm{~s}$ for all the statistics.

When using the BT Tower data, it is not feasible for the LES to model flows and dispersion at full-scale Reynolds numbers, due to the current computer capability. Instead the BT Tower data were scaled down to the scale of wind-tunnel model, i.e. 1:200 for length and 1:2 for velocity (with viscosity un-scaled). So the 
model/field time-scale ratio was 1:100. This also means that the scaled-down Reynolds number $U_{r e f} h_{m} / v$ was approximately 18,000 , where $U_{r e f}$ was the scaled mean velocity at the BT Tower top, $h_{m}$ was scaled mean building height. For the purpose of easy reading, some numerical settings and results are described in full scale in the paper. For example, the LES was initialized at 1600 on 3 June 2004 (in full scale, see Fig. 6). The source was switched on at 1630 when the sampling was started and turned off at 1645, but the sampling continued until 1700.

\section{Flows and Dispersion in $-90^{\circ}$ and $-51^{\circ}$ Winds}

The $-51^{\circ}$ wind, approximately a south-west wind, is a typical oblique wind. The flow and dispersion results in this wind are shown and discussed in Carpentieri et al. (2009) and Xie and Castro (2009). The $-90^{\circ}$ wind, approximately a southerly wind, is perpendicular to Marylebone $\mathrm{Rd}$ and also to the windward surfaces of most of the buildings. The DAPPLE streets (see Fig. 1a) are largely organised along $0^{\circ}$ and $90^{\circ}$ directions. Hence in the $-90^{\circ}$ wind the street flows are likely to be dominated by channelling along the streets and recirculations in the perpendicular streets, whereas in the $-51^{\circ}$ wind the branching of the flow around the buildings, which leads to the so-called topological dispersion, is likely to be important. These are intensively investigated, discussed or reviewed in literature for flows and dispersion over a group of regular obstacles, for example, Davidson et al. (1996), Belcher (2005), Claus et al. (2009) and Branford et al. (2011). However, the geometry of the DAPPLE site is more complicated than 'regular'. For instance, there are a few tall buildings, which may have significant effects on flows (Xie et al., 2008) and dispersion. The buildings on the north side of Marylebone Rd are not well aligned (Fig. 1a); flow pattern may not be insensitive to this mis-alignment. Certainly it is of interest to perform a quantitative comparison of flows and dispersion in the $-90^{\circ}$ and $-51^{\circ}$ winds over the realistic urban geometry, i.e. the DAPPLE site.

It might not be too surprising to find that (both numerically and experimentally) the modelled turbulence at the major intersection is more sensitive to the artificial boundary conditions at the leading edges of the DAPPLE site (i.e. the region with blue and green colour in Fig. 2) for the $-90^{\circ}$ wind than for the $-51^{\circ}$ wind. Firstly, the upwind fetch in the 1:200 model is longer for the $-51^{\circ}$ wind than for the $-90^{\circ}$ wind. Secondly, in the $-51^{\circ}$ wind the branching of the flow around buildings is likely important, whereas in the $-90^{\circ}$ wind the channelling along the streets, e.g. Gloucester $\mathrm{Pl}$, is dominant.

Again it has been found that wind the direction effect on the spatially averaged turbulent statistics over a group of cubes is significant (Claus et al., 2009). The spatial average of the magnitude of the time-mean velocity $\left(U_{\text {mag }}\right)$ and resolved turbulent kinetic energy $\left(T K E=\frac{1}{2} \overline{u_{i}^{\prime} u_{i}^{\prime}}\right)$ within and immediately above the urban canopy in the $-51^{\circ}$ and $-90^{\circ}$ winds are plotted in Fig. 3a and $\mathrm{b}$. These were obtained by averaging over a region $-1000 \mathrm{~mm}<x_{t} \leq 1000 \mathrm{~mm},-1000 \mathrm{~mm}<y_{t} \leq 1000 \mathrm{~mm}$, which covers most of the DAPPLE site and has a packing density about 0.53 , slightly greater than that of the whole DAPPLE site. The two sets of profiles show a similar pattern. The peaks of $T K E$ and magnitude of Reynolds shear stress $\left(R S T_{m}=\right.$ $\sqrt{{\overline{u^{\prime} w^{\prime}}}^{2}+{\overline{v^{\prime} w^{\prime}}}^{2}}$, not shown here) occur at height $1.5 h_{m}$, not at the mean building height $h_{m}$. This is probably due to the five large and tall buildings with heights around $1.5 h_{m}$ as discussed in Xie et al. (2008) and Xie and Castro (2009). This also suggests that the peaks occur at the height of the tallest buildings regardless of the wind direction. The predicted TKE and magnitude of Reynolds shear stresses within and immediately above the canopy in the $-90^{\circ}$ wind are slightly greater than those in the $-51^{\circ}$ wind. The greater TKE and stronger shear in the $-90^{\circ}$ wind are likely due to the large recirculations in the perpendicular streets. The predicted velocity magnitude in the $-90^{\circ}$ wind is less than that in the $-51^{\circ}$ wind. Overall, the difference in 

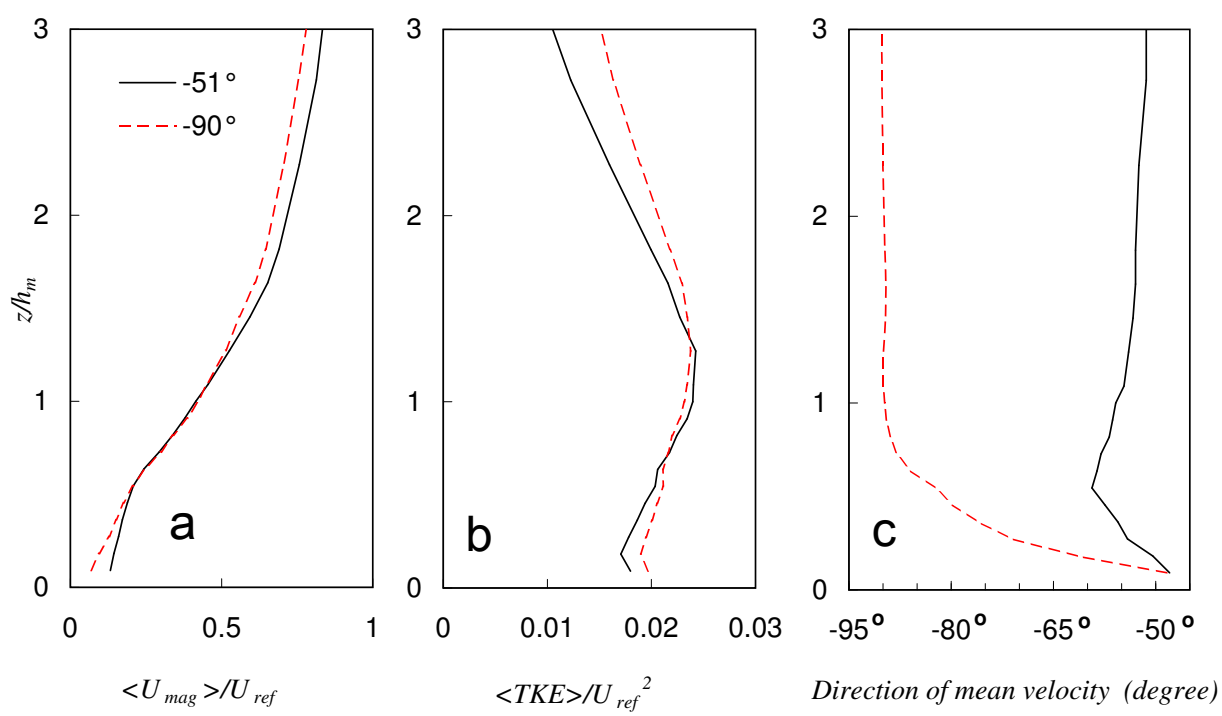

Fig. 3 Vertical profiles: (a) spatial average of the magnitude of the time-mean velocity, (b) resolved turbulent kinetic energy and (c) direction of the spatial average of the time-mean velocity (i.e. bearing clockwise to the Marylebone Rd direction).

the magnitude of the spatially-averaged quantities between the two wind directions is not as significant as might have been anticipated.

Fig. $3 c$ shows vertical profiles of the direction of the spatial average of the time-mean velocity within and immediately above the canopy. For the $-51^{\circ}$ wind, the velocity direction changes no more than $10^{\circ}$ within the canopy. In contrast, for the $-90^{\circ}$ wind the velocity direction turns clockwise from $-90^{\circ}$ above the canopy to about $-50^{\circ}$ within the canopy. It is to be noted that all the LES computations here were driven by constant mass flow rates at the inlet boundaries, rather than a constant pressure gradient as used by Claus et al. (2009) who report that the wind direction changes significantly within the canopy.

It is very interesting that the velocity direction near ground level is about $-50^{\circ}$ for both the forcing directions. In order to check that this was not caused by the boundary-condition settings, a second simulation with different boundary conditions was performed as detailed in Section 3. No significant difference in the direction of the spatially averaged velocity was observed. To further interpret the directional change of the spatially averaged velocity within the canopy in the $-90^{\circ}$ wind, mean velocity vectors $\left(U_{m}, V_{m}\right)$ on horizontal planes at different heights (i.e. $z / h_{m}=0.1,0.5$ and 1) over Marylebone Rd are plotted in Fig. 4. Fig. $4 \mathrm{a}$ and $\mathrm{b}$ show a strong west-to-east rather than east-to-west flow along Marylebone Rd. This is likely due to the local arrangement of the buildings, i.e. the three slightly staggered buildings off Marylebone Rd, which are marked with 'staggered' in Fig. 4a. For example, the sudden increase of the street width at the east side of the major intersection induces air from Gloucester Pl to flow west-to-east. Also at the Balcombe St - Marylebone Rd and Baker St -Marylebone Rd intersections, similar flow patterns are observed which are likely due to the same mechanics. These at the three intersections together enhance forcing the in-street wind to go west-to-east in the $-90^{\circ}$ wind. Such phenomena were also observed in the wind-tunnel experiments. And the field experiments show that for the near $\pm 90^{\circ}$ roof winds, the in-street wind at the Marylebone-Rd stations near the major intersection was more probably west-to-east than east-west (Balogun et al., 2010). It is also to be noted that Marylebone Rd is nearly $30 \%$ of the total street area in the region $-1000 \mathrm{~mm}$ 


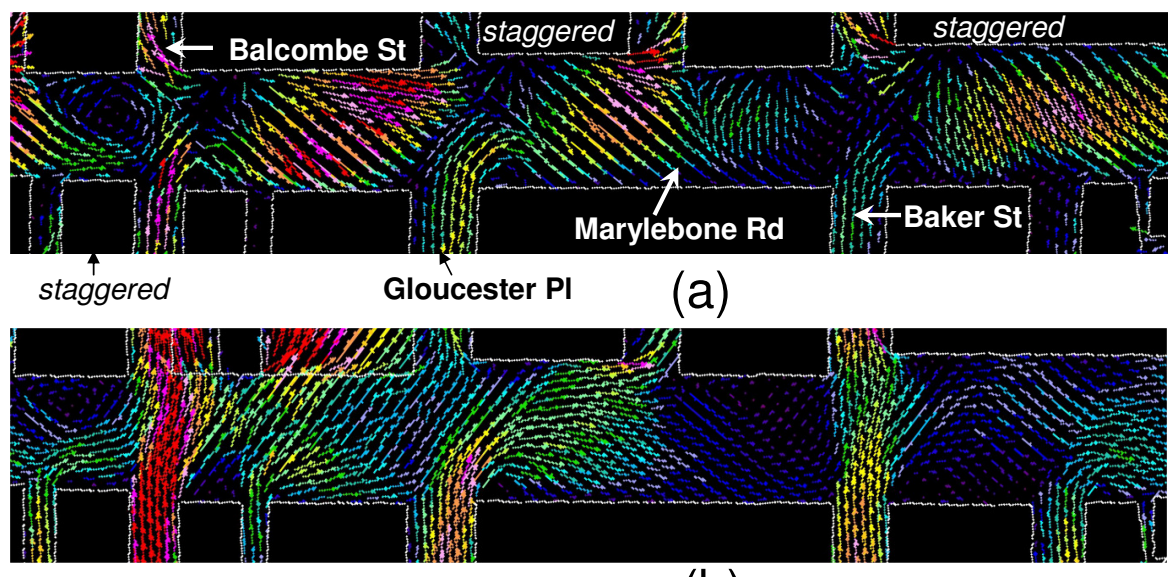

(b)

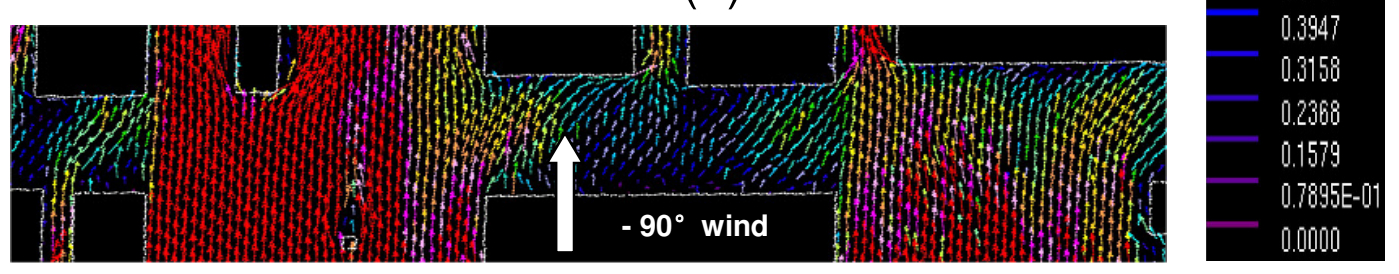

(c)

Fig. 4 Mean velocity vectors $\left(U_{m}, V_{m}\right)$ on horizontal planes over Marylebone Rd at different heights in $-90^{\circ}$ wind. (a) $z / h_{m}=0.1$; (b) $z / h_{m}=0.5$; (c) $z / h_{m}=1.0$. Image resolution for the vectors is $20 \mathrm{~mm}$ in WTM scale (4 $\mathrm{m}$ in full scale).

$<x_{t}, y_{t} \leq 1000 \mathrm{~mm}$. Hence the flow along Marylebone Rd provides a great contribution to the spatially averaged velocity in this region. This may largely explain the variation of the profile in the $-90^{\circ}$ wind in Fig. 3c.

The non-reactive tracer was released at station $S 2\left(x_{t}=-356 \mathrm{~mm}, y_{t}=-702 \mathrm{~mm}, \mathrm{z}=10 \mathrm{~mm}\right.$ in Fig. 1a). LES of dispersion in the $-51^{\circ}$ wind was validated in our previous work (Xie and Castro, 2009) using wind-tunnel data. A few measurements were also obtained for the validation of dispersion in the $-90^{\circ}$ wind (see Fig. 5a). Fig. 5 plots profiles of mean concentration $C_{m}$ and concentration fluctuation $c_{r m s}$ along Marylebone Rd (i.e. $y_{t}=0$ ) at various heights in the $-51^{\circ}$ and $-90^{\circ}$ winds. The LES slightly over-predicted the mean concentration compared with the measurements. But this is no worse than the validation for the $-51^{\circ}$ wind conditions (Xie and Castro, 2009). The centre of an ideal Gaussian plume released from the source $S 2$ and heading in the $-51^{\circ}$ direction will intersect Marylebone Rd approximately at $x_{t}=200 \mathrm{~mm}$. However, Fig. 5a shows that the maximum concentration in the $-51^{\circ}$ wind occurs at $x_{t}=140 \mathrm{~mm}$. Given that clear air was convected along Marylebone $\mathrm{Rd}$ from the upwind region (see Fig. 5a), this might suggest a strong branching of the flow and concentration cloud around the buildings, in particular along York St Gloucester Pl - Marylebone Rd. The secondary peaks approximately at $x_{t}=400 \mathrm{~mm}$ for the profiles at $z=$ $55 \mathrm{~mm}$ and $z=110 \mathrm{~mm}$ in the $-51^{\circ}$ wind might be because the plume is not significantly 'non-Gaussian' and that station is not too far from the centre of the plume. No secondary peak was found at $x_{t}=400 \mathrm{~mm}$ for 

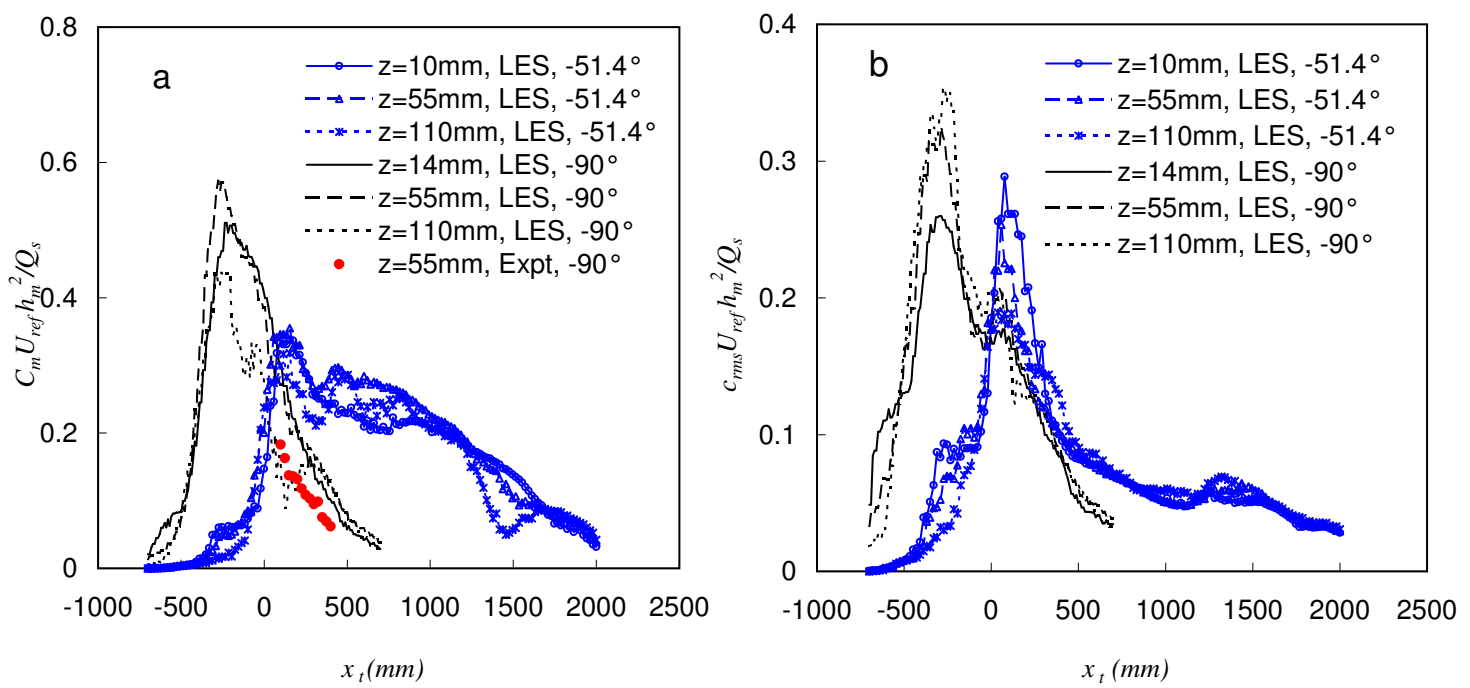

Fig. 5 Profiles of (a) $C_{m}$ and (b) $c_{r m s}$ along Marylebone Rd (i.e. $y_{t}=0$ ) in the $-51^{\circ}$ wind and $-90^{\circ}$ winds. Abscissa in WTM scale.

the profile at $z=10 \mathrm{~mm}$. A long tail on the downwind side the profiles in the $-51^{\circ}$ wind suggests a strong along-street plume in Marylebone Rd. In contrast, the profiles of mean concentration in the $-90^{\circ}$ wind are more symmetrical than those in the $-51^{\circ}$ wind, for which the maxima occur approximately at $x_{t}=-250$ $\mathrm{mm}$, which is near the centre of an ideal Gaussian plume released from the source $S 2$ in the $-90^{\circ}$ wind. This suggests that the across plume component is more dominant than the channelling plume component for the $-90^{\circ}$ wind and the source $S 2$. It is to be expected that the plume width is greater in the $-51^{\circ}$ wind than that in the $-90^{\circ}$ wind, since the $-51^{\circ}$ wind is more in favour of the plume spreading along the chessboard-like streets due to the branching.

Fig. 5b shows more significant peaks in the $c_{r m s}$ profiles at the major intersection than those of the $C_{m}$ profiles in the $-51^{\circ}$ wind. This is because of the strong intermittency of the concentration at the major intersection, which also means that the maximum instantaneous concentration at the major intersection must be much greater than the mean. The $c_{r m s}$ profiles in the $-90^{\circ}$ wind show secondary peaks at the major intersection, and also show a less symmetrical form than the $C_{m}$ profiles in the same wind. This indicates a strong intermittency of the concentration at the major intersection in the $-90^{\circ}$ wind too. Overall, the effect of wind direction on near field dispersion is significant.

\section{Using Realistic Wind Conditions to Drive Street-Scale LES}

In principle it is possible to use the data generated by mesoscale meteorological models, e.g. the UK Met Office's Unified Model (UM) or the NCAR's Weather Research \& Forecasting (WRF) model, as boundary 


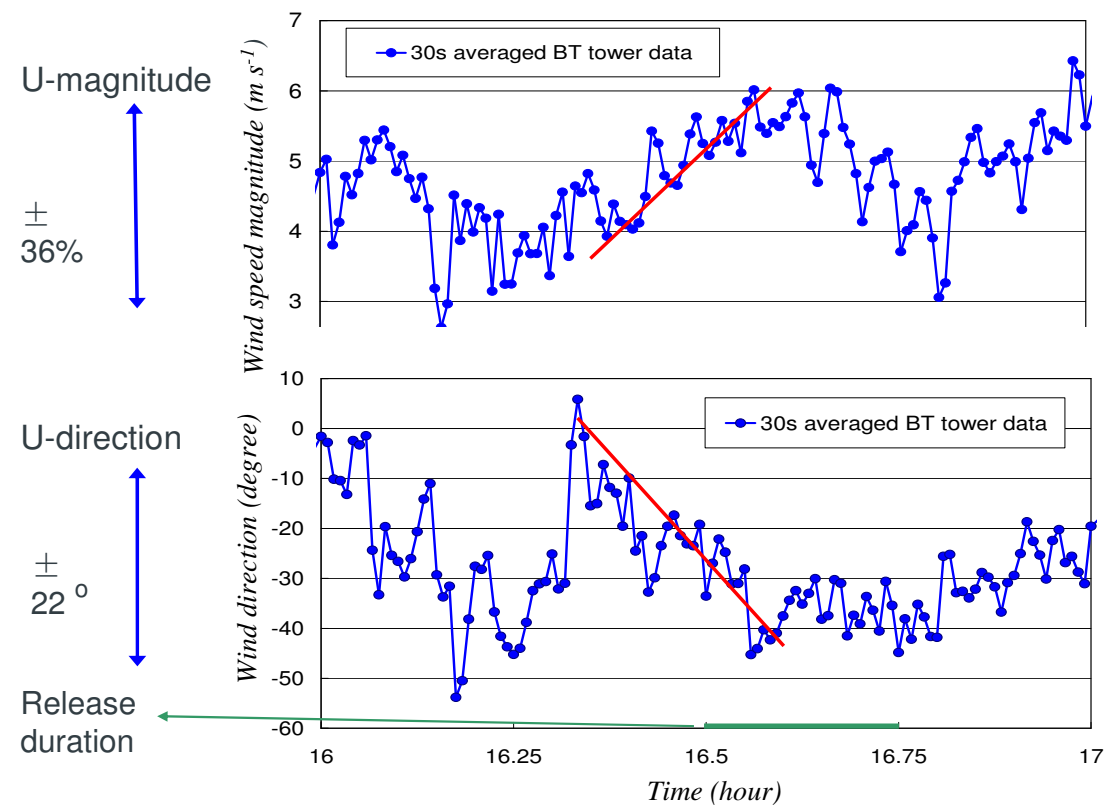

Fig. 630 -sec averaged wind magnitude $U$ and direction $\theta$ (bearing clockwise to the Marylebone-Rd direction, i.e. $x_{t}$ in Fig. 1) at the BT Tower top with a mark indicating 15-min release duration.

conditions to drive street-scale LES. Again, it is still a great challenge to use these data directly at present. For example, the time interval of UM data stored at the British Atmospheric Data Centre (BADC) is one hour, which is about two decades greater than the integral time scale (see Fig. 7). How to fill this gap is a big issue yet to be resolved.

$10 \mathrm{~Hz}$ resolution wind data were measured at $190 \mathrm{~m}$ above street level on the BT Tower (Wood et al., 2009), which was approximately $1500 \mathrm{~m}$ east of the DAPPLE site. The data were processed as 60-sec and 30 -sec averaged data. Fig. 6 plots the magnitude and direction of the 30-sec averaged horizontal velocity during 1600-1700 (with a mark indicating the source release duration 1630-1645) on 3 June 2004. Again the wind direction is defined as the angle bearing clockwise to the Marylebone-Rd direction, i.e. $x_{t}$ in Fig. 1. As a test case, these data were used to generate inflow conditions to drive the large-eddy simulations. Again it is to be noted that the LES was initialized at 1600 with the source release switched on at 1630 and off at 1645 , and with the sampling and averaging started at 1630 until 1700. It was noticed that in this duration the wind speed magnitude varied $\pm 36 \%$ and the wind direction varied $\pm 22^{\circ}$. In the release duration 1630-1645 and also afterwards up to 1700, the wind direction was fairly steady, almost south-west. Before the release of the tracer, the swing of wind direction was great, from $0^{\circ}$ to $-40^{\circ}$ in less than 15 minutes.

Fig. 7 shows the spectrum of the times series of wind direction $\theta$ and horizontal velocity magnitude $U$ measured at a $10-\mathrm{Hz}$ sampling rate at the BT Tower top. The turbulence integral time scale is approximately 1 minute. The LES timestep $\Delta t$ is at the high-frequency end of the inertial subrange and is about two decades less than the integral scale. It is to be noted that the BT Tower data with frequencies higher than the update frequency (e.g. $1 / \delta t=1 / 30 \mathrm{~Hz}$ ) were not filtered for use to calculate the spectrum in Fig. 7. A different update frequency $1 / 60 \mathrm{~Hz}$ was also tested as a numerical experiment, and no significant effect was found. Turbulence fluctuations with frequencies greater than the update frequency were reproduced using 


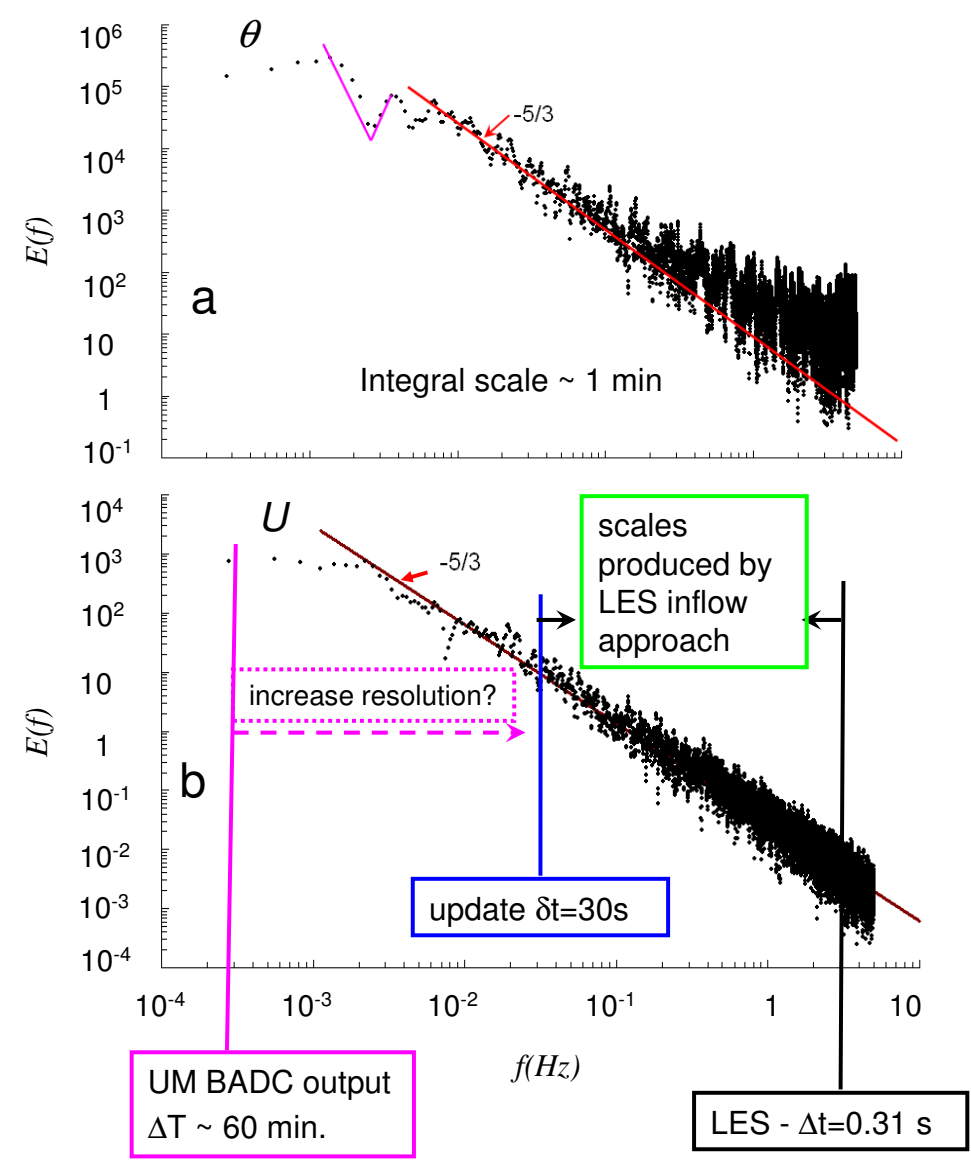

Fig. 7 Spectrum of time series measured at a 10-Hz sampling rate at the BT Tower top. (a) wind direction $\theta$ and (b) horizontal velocity magnitude $U$. UM, UK Met Office's Unified Model; BADC, the British Atmospheric Data Centre.

our inflow generator (Xie and Castro, 2008). This is because measured instantaneous turbulence fluctuations are not always available, and indeed extremely sparse and expensive to obtain. It is to be noted again that the time interval of the UM data stored at the British Atmospheric Data Centre (which are available for users to download,) is about two decades greater than the integral time scale. Currently the timestep of the mesoscale meteorological models, e.g. the UM, is in the order of 1 minute. However, it is not clear how much of the motion in that scale is smoothed out in these models.

Fig. 8 presents mean wind speed along Marylebone $\mathrm{Rd}\left(y_{t}=0\right)$ and Gloucester $\mathrm{Pl}\left(x_{t}=0\right)$ in realistic wind conditions (i.e. 'BT Tower data') and steady wind conditions (i.e. 'Mean wind'). For the latter, the averaged wind velocity magnitude and direction of the BT Tower data from 1630 to 1700 were taken as the velocity magnitude and direction for the inflow generation. It is to be noted that the inflow approach was used to generate artificial turbulence at the three inlets for all of the LES computations. There is a degree of discrepancy between the two profiles. It seems that the discrepancy in Fig. 8a is slightly greater than that in Fig. 8b. This indicates that the mean flow field over Marylebone Rd was more sensitive to the variation of 

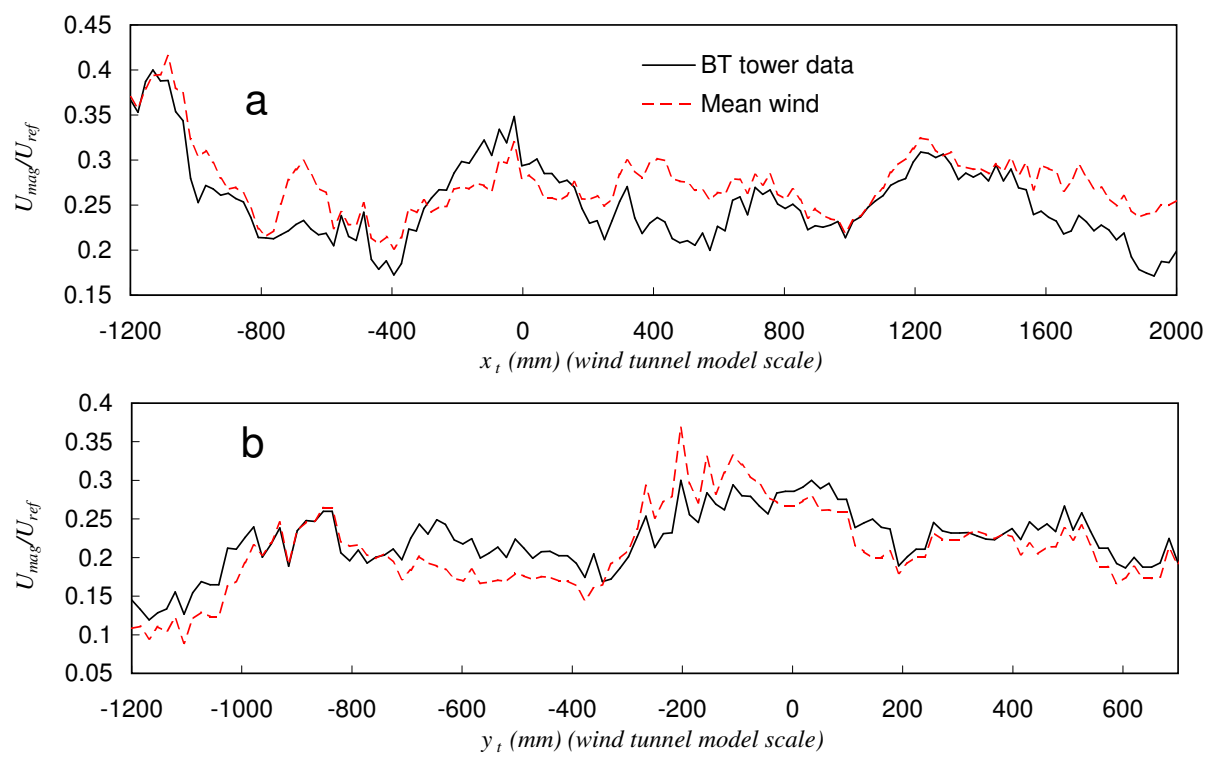

Fig. 8 Mean wind speed at height $z / h_{m}=0.5$ along (a) Marylebone $\mathrm{Rd}\left(y_{t}=0\right)$ and (b) Gloucester Pl $\left(x_{t}=0\right)$, in various wind conditions. Abscissa in WTM scale.

Table 1 Coordinates in wind-tunnel model (Fig. 1a) of the corresponding field stations (Fig. 1b)

\begin{tabular}{lrrrrrrr}
\hline Site & $X_{2}$ & 2 & 4 & 6 & 8 & 12 & 13 \\
\hline$x_{t}(\mathrm{~mm})$ & -1125 & -500 & -288 & 200 & 500 & 1793 & -72 \\
$y_{t}(\mathrm{~mm})$ & -740 & -350 & -365 & 100 & -100 & -82 & -240 \\
$z(\mathrm{~mm})$ & 10 & 10 & 10 & 10 & 10 & -269 \\
\hline
\end{tabular}

wind direction and speed than over Gloucester Pl, which is probably because the buildings off Marylebone $\mathrm{Rd}$ were more irregular and also the street width was greater. The variation of the wind direction is likely the more important factor than that of the wind speed. However, the overall correlation of the two profiles is great, which may suggest that the effect on the time-averaged velocity magnitude of the variation of wind conditions was not extremely significant in this particular case.

The point source $X 2$ and 16 sampling stations of the DAPPLE field experiments on 3 June are shown in Fig. 1b. Table 1 shows the coordinates in the wind-tunnel model (also see Fig. 1a) of the point source $X 2$ and 7 sampling sites, at which the field and LES data were compared.

Fig. 9 shows a comparison of the 3-min averaged concentration at site 14 (the Westminster Council House doorway on west side of the Gloucester Pl by the major intersection) between the field measurements and the six sets of LES results in different wind conditions. The two cases 'LES run1, BT tower data' and 'LES run2, BT tower data' using the BT tower data for the inflow generation were initialized from different initial conditions. Both of the LES results are in fairly good agreement with measurements, which suggests that the 3-min averaged concentration was not significantly sensitive to the initial conditions of the largeeddy simulations. The wind speed and direction of the case 'LES, mean wind' in steady wind conditions were equivalent to the mean wind speed $\langle U\rangle$ and the mean direction $\langle\theta\rangle$ of the BT Tower data from 1630 to 1700 . The three cases 'LES, mean wind $+6^{\circ}$, ' $\mathrm{LES}$, mean wind $+12^{\circ}$ ' and 'LES, mean 


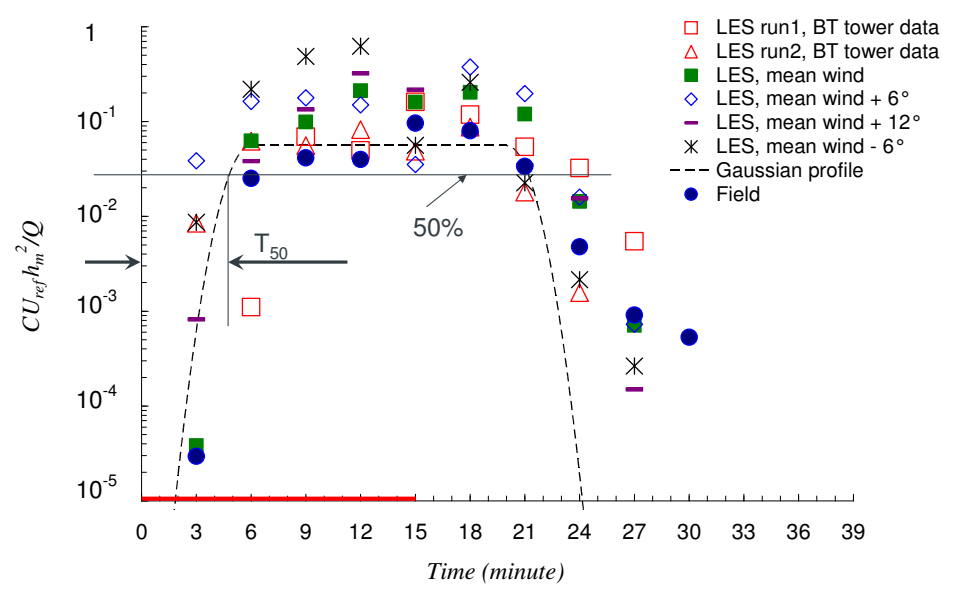

Fig. 9 3-min averaged concentration at site 14 in different wind conditions. Abscissa in full scale. $U_{\text {ref }}$, free-stream velocity; $Q$, concentration flux at source; $h_{m}$, mean building height; $T_{50}$, advection time. Lines: red solid, release duration; dashed, approximate location and width of a plume in combined Gaussian form; thin black solid, 50\% level of maximum of the Gaussian profile. Symbols: $\bullet$, field measurements; $\square$ and $\triangle$, using the BT Tower data (Fig. 6) for inflow generation with different initial conditions; $\square$, $\diamond,-, *$, using steady velocity magnitude and directions (for inflow generation) equivalent to $\left\langle U>\right.$ and $\left\langle\theta>,\left\langle\theta>+6^{\circ},\left\langle\theta>+12^{\circ}\right.\right.\right.$ and $\langle\theta\rangle-6^{\circ}$ respectively, where $\langle U\rangle$ is the mean wind speed and $\langle\theta\rangle$ is the mean direction of the BT Tower data from 1630 to 1700 .

wind $-6^{\circ}$, in steady wind conditions, for which the wind speeds were $\langle U\rangle$ and the wind directions were $\left\langle\theta>+6^{\circ},\left\langle\theta>+12^{\circ},\left\langle\theta>-6^{\circ}\right.\right.\right.$ respectively, were investigated to check the sensitivity of wind direction on dispersion. Not surprisingly, Fig. 9 shows that the 3 -min averaged concentration in the steady winds is sensitive to the wind direction. It was also noticed that the calculated peak mean concentration in the steady wind conditions can be one order of magnitude greater than those numerical results in the realistic wind conditions and those field measurements. One might argue that it is unfair to make a quantitative comparison of the 3-min averaged concentration at one site between the field experiment (with a single 15-min release) and the LES computations, because of the relatively short averaging. However, the data at the other six sites (see Table 1, Figs. 1b and 10) collectively confirmed these speculations. So it is concluded that LES in steady wind conditions over-predicted the peak concentration, whereas LES in realistic wind conditions produced significantly improved results compared with the field measurements.

The 3-min averaged concentration time series of the two cases 'LES run1, BT tower data' and 'LES run2, BT tower data' were fitted into a combined profile with two symmetric 'half Gaussian profiles' at left and right ends and a constant profile in the middle, where the constant is the maximum of the Gaussian profiles. Following Cheng and Robins (2004), $T_{50}$ is defined as the time duration for the plume front with a half value of the local maximum concentration to reach a station, which measures the advection time from the source to the station. The advection velocity was estimated using

$$
U_{a d v}=\frac{D}{T_{50}}
$$

where $D=\sqrt{\left[x_{t}(\text { site })-x_{t}(X 2)\right]^{2}+\left[y_{t}(\text { site })-y_{t}(X 2)\right]^{2}}$ is the distance from the site to the source $X 2$. Here the $T_{50}$ for site 14 was estimated to be approximately 5 minutes and the advection velocity $U_{a d v}$ is about 


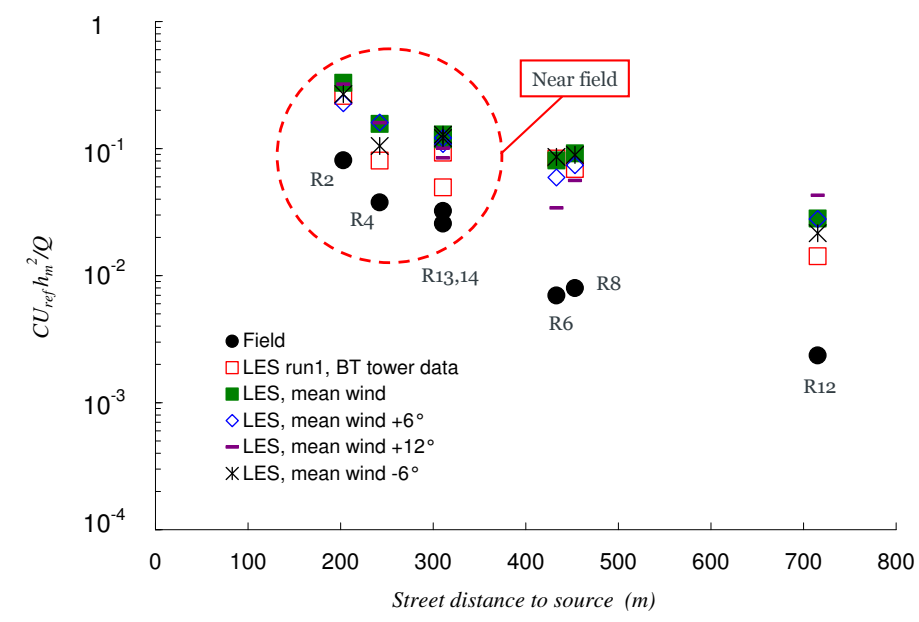

Fig. 10 30-min averaged concentration at 7 sites. Abscissa in full scale. $U_{\text {ref }}$, free-stream velocity; $Q$, concentration flux at source; $h_{m}$, mean building height. Symbols: •, field measurements; $\square$, using the BT Tower data (Fig. 6) for inflow generation; $\mathbf{\square}, \diamond,-, *$, using steady velocity magnitude and directions (for inflow generation) equivalent to $\left\langle U>\right.$ and $\left\langle\theta>,\left\langle\theta>+6^{\circ},\left\langle\theta>+12^{\circ}\right.\right.\right.$ and $\langle\theta\rangle-6^{\circ}$ respectively, where $\langle U\rangle$ is the mean wind speed and $\langle\theta\rangle$ is the mean direction of the BT Tower data from 1630 to 1700 .

$0.15 U_{\text {ref }}$, which is close to the value $0.16 U_{\text {ref }}$ suggested in Cheng and Robins (2004) who performed windtunnel experiments in steady wind conditions. This suggests that the advection velocity in a varying wind is not too different from that in a steady wind. It is to be noted that at the right end of the profiles, most of the LES and field data show a longer tail than at the left side. This is not surprising and is due mainly to the complex urban geometry. Source material tends to be trapped in the canopy and is entrained out relatively slowly. Again this is the so-called secondary source effect. It is stressed in Section 1 that the large time-scale fluctuations of the wind direction enhance the effect of the secondary sources (Belcher, 2005). A similar but likely weaker effect is also expected over open countryside where the wind profile means that a small amount of material near the ground is advected very slowly.

Fig. 10 presents a comparison of 30-min averaged concentration at the 7 sites obtained in the field experiments and in the LES computations, against street distance to the source $X 2$, which is defined as $\mid x_{t}($ site $)-x_{t}(X 2)|+| y_{t}($ site $)-y_{t}(X 2) \mid$. The field data at the other 9 sites were incomplete and thus could not be used here. The averaging was started when the tracer was released. Thirty minutes after the start of the release, the concentration at the 7 sites was found negligible compared with the 30-min mean (see Fig. 9). It is to be noted that the 7 sites were all essentially in the core of the plume. We emphasize that compared with the field measurements the LES using the BT Tower data significantly improved the prediction at the near field sites, i.e. sites 2, 4, 13 and 14, than all of those LES computations and wind-tunnel experiments in steady wind conditions (also see Cheng and Robins, 2004; Xie and Castro, 2009). Nonetheless, at the far field sites 6,8 and 12, all of the numerical simulations seriously over-predicted the mean concentration. This is most likely due to the thermal convection not included in the LES model or the wind tunnel-model. During 1600-1700 on 3 June 2004, London urban area was in weakly unstable conditions with a Richardson number around -0.1 (based on the shear stress, heat flux, mean temperature and mean velocity measured at the BT Tower top and the mean building height, Wood et al., 2010). More possible sources of discrepancy, e.g. the effects of domain height, are discussed in the following paragraphs. 

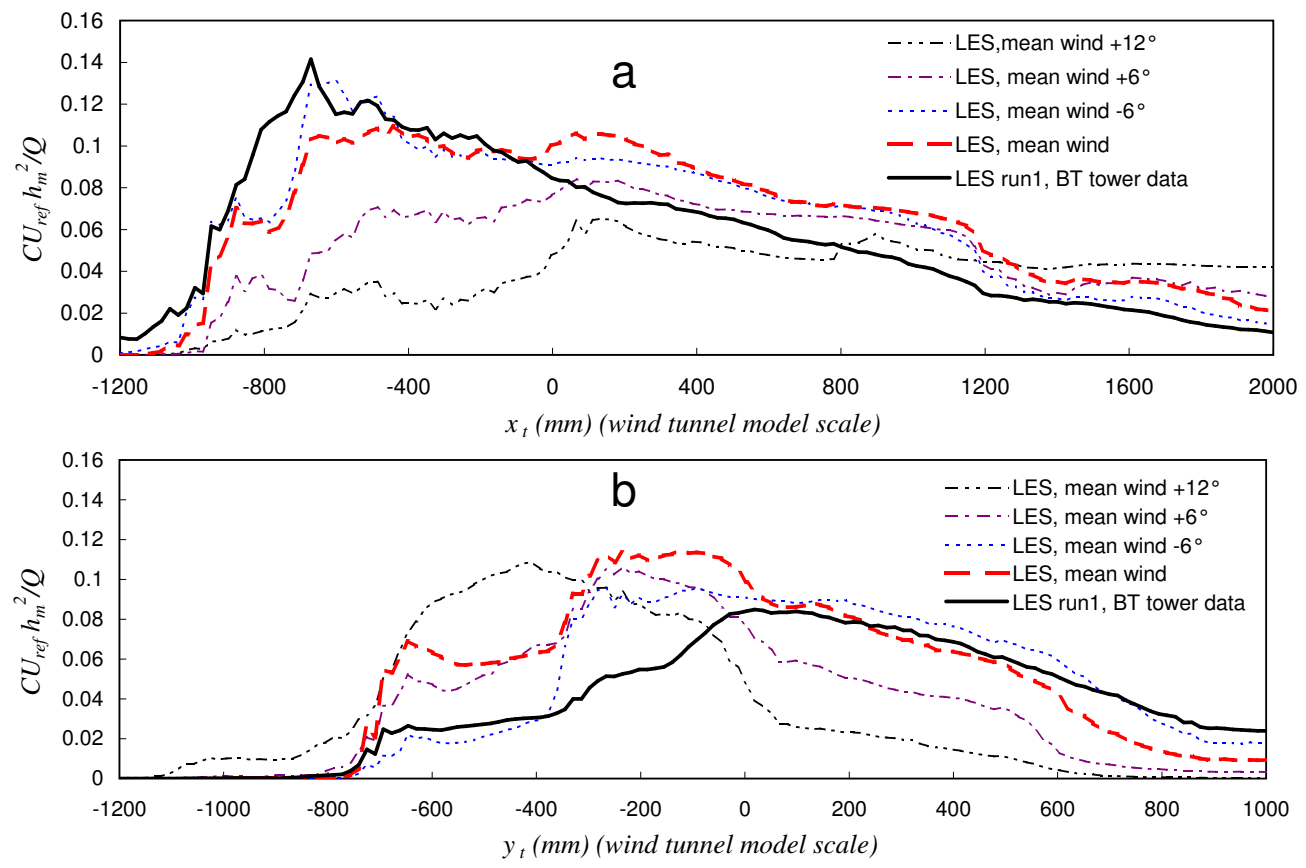

Fig. 11 30-min averaged concentration under various wind conditions at $z / h_{m}=0.13$ along (a) Marylebone Rd ( $y_{t}=0$ ) and (b) Gloucester Pl $\left(x_{t}=0\right)$. Abscissa in WTM scale. $U_{r e f}$, free-stream velocity; $Q$, concentration flux at source; $h_{m}$, mean building height. Lines: _ - using the BT Tower data (Fig. 6) for inflow generation; - - , - - - , - - - and $\cdots$, using steady velocity magnitude and directions (for inflow generation) equivalent to $\langle U\rangle$ and $\langle\theta\rangle,\langle\theta\rangle+12^{\circ},\langle\theta\rangle+6^{\circ}$ and $\langle\theta\rangle-6^{\circ}$ respectively, where $\langle U\rangle$ is the mean wind speed and $\langle\theta\rangle$ is the mean direction of the BT Tower data from 1630 to 1700 .

Fig. 11a shows LES-predicted 30-min averaged concentration profiles at height $z / h_{m}=0.13$ along Marylebone $\mathrm{Rd}\left(y_{t}=0\right)$ in the five wind conditions. Because of the variation of the wind direction for the case 'LES run 1, BT tower data', probably more scalar is transported through the near-source south-north streets into Marylebone $\mathrm{Rd}$ than that in the steady wind conditions, and this significantly increases the mean concentration in the range $-800 \mathrm{~mm} \leq x_{t}<-500 \mathrm{~mm}$. It is to be noted in Fig. 10 all of the numerical simulations over-predicted the mean concentration at the 7 sites to various extents. In Fig. 11a apart from the region upstream of the major intersection (i.e. $x_{t}<0$ ), the mean concentration of the case 'LES run 1, BT tower data' is evidently lower than those in the cases 'LES, mean wind' and 'LES, mean wind $\pm 6^{\circ}$, which confirms again that the large-eddy simulations in realistic wind conditions improve the predictions.

There is an evident increase of mean concentration at the major intersection $\left(x_{t}=0, y_{t}=0\right)$ for all of the simulations in the steady winds (Fig. 11a). This is certainly due to large concentration clouds transported from Gloucester Pl (see Fig. 11b for those in steady winds and Fig. 12c). In contrast, no such increase is observed at all for case 'LES run 1, BT tower data', which might be due to the great concentration in the range $-800 \mathrm{~mm} \leq x_{t}<-500 \mathrm{~mm}$ along Marylebone $\mathrm{Rd}$ as discussed earlier. The evident increase of mean concentration in the steady winds also suggests that mixing at the major intersection is less efficient than that in the varying-direction wind. One major mechanism for this is that the fluctuations of wind direction 
enhances the branching of the flow around buildings, which leads to stronger topological dispersion than in the steady winds. For the 'LES, mean wind $+6^{\circ}$ ' and 'LES, mean wind $+12^{\circ}$ ' cases, Marylebone $\mathrm{Rd}$ is nearer to the edge of the plume. Fig. $11 \mathrm{~b}$ confirms that with increasing angle of the wind direction, the plume centre shifts further upstream along Gloucester Pl.

Fig. 11b plots LES-predicted 30-min averaged concentration profiles at height $z / h_{m}=0.13$ along Gloucester Pl $\left(x_{t}=0\right)$. The mean concentration in the realistic wind 'LES run 1, BT tower data' is significantly less than that in the three steady winds, 'LES, mean wind', 'LES, mean wind $+6^{\circ}$ ' and 'LES, mean wind $+12^{\circ}$, in the range $-800 \mathrm{~mm} \leq x_{t}<-100 \mathrm{~mm}$, and that in the steady wind 'LES, mean wind $-6^{\circ}$ in the range $-400 \mathrm{~mm} \leq x_{t}<-100 \mathrm{~mm}$. Also the maximum mean concentration of the plume in the realistic wind 'LES run 1, BT tower data' is evidently less than that in the steady wind 'LES, mean wind', which suggests a greater plume width for the former. Again, this is because the variation of the wind direction enhances the dispersion, in particular the topological dispersion. In contrast, in the range $y_{t}>$ $-100 \mathrm{~mm}$ there is no significant difference between the three winds, 'LES run 1, BT tower data', 'LES, mean wind' and 'LES, mean wind $-6^{\circ}$ '. This again confirms that the mean concentration in the near field was essentially more sensitive to the variation of wind direction than that in the far field. With respect to the other two steady winds, 'LES, mean wind $+12^{\circ}$ ' and 'LES, mean wind $+6^{\circ}$ ', the sampling stations in the range $y_{t}>-100 \mathrm{~mm}$ along Gloucester Pl were too far from the core of the plume and subsequently it is probably not reasonable to compare the mean concentration in these two wind conditions with that in the other wind conditions.

Compared with the field measurements, the large-eddy simulations using the realistic wind conditions significantly improved the prediction at the near-field sites. One of the reasons was because the variation of wind direction enhanced the topological dispersion, as discussed earlier. However, at the far-field sites, the prediction was only marginally improved. Why is this? Fig. 12a and b show conceptual sketches of the effects on dispersion of long-time variations of wind direction. It is assumed that the driving-wind direction $\theta$ is zero up to time $t$ and the velocity component in the direction $y_{t}$ is zero (Fig. 12a). In a short time (e.g. the update timestep $\delta t=30 \mathrm{~s}$ as shown in Fig. 7) the direction of the driving wind changes to an oblique direction for a short duration (e.g. $\delta t$ ) due to a weather-scale eddy passing over the domain (Fig. 12b). A uniform velocity component $U \sin \theta$ in the direction $y_{t}$ is assumed over the whole domain provided that the eddy is large enough. Hence a translational lateral displacement of the plume is expected - the same lateral displacement for the far-field and near-field plumes (except for the immediately-near-source plume which is denoted by dashed-dot lines in Fig. 12). Fig. 12b shows that the near-field station is at the edge of the plume, whereas the far-field station is still near the centre of the plume. Fig. 12a and b might be analogous with the dispersion from a point source which oscillates in the transverse direction. These suggest that the near field is bound to be affected much more than the far field by long-time variations of wind direction. Also a wider plume in the near field is expected in a fluctuating wind than in a steady wind, whereas a smaller difference is expected in the far field. Note that in urban environments, the dispersion is also strongly affected by the urban topography, in particular in the near field.

In order to check the above speculation, a comparison of 30-min averaged concentration contours at height $z / h_{m}=0.9$ under two wind conditions is shown in Fig. 12c and d. Fig. 12c shows LES results in the steady-wind conditions in which the 30-min-averaged wind magnitude $U$ and direction $\theta$ of the BT Tower data (from 1630 to 1700 on 3 June) were used to generate the inflow conditions. Fig. 12d shows LES results in the unsteady wind conditions, which are the 30-sec-averaged BT Tower data shown in Fig. 6. Note that the colour bar in Fig. 12 is for a comparison purpose only between $\mathrm{c}$ and $\mathrm{d}$. The plume width (see the four marks ' $I$ ' in the figure) of the near field in d is significantly greater than that in c. However, in the far field the plume width is just marginally greater in $\mathrm{d}$ than that in $\mathrm{c}$. Also note that the concentration at the major intersection in the near field in $\mathrm{c}$ is much greater than that in $\mathrm{d}$. But the difference in the concentration in the 

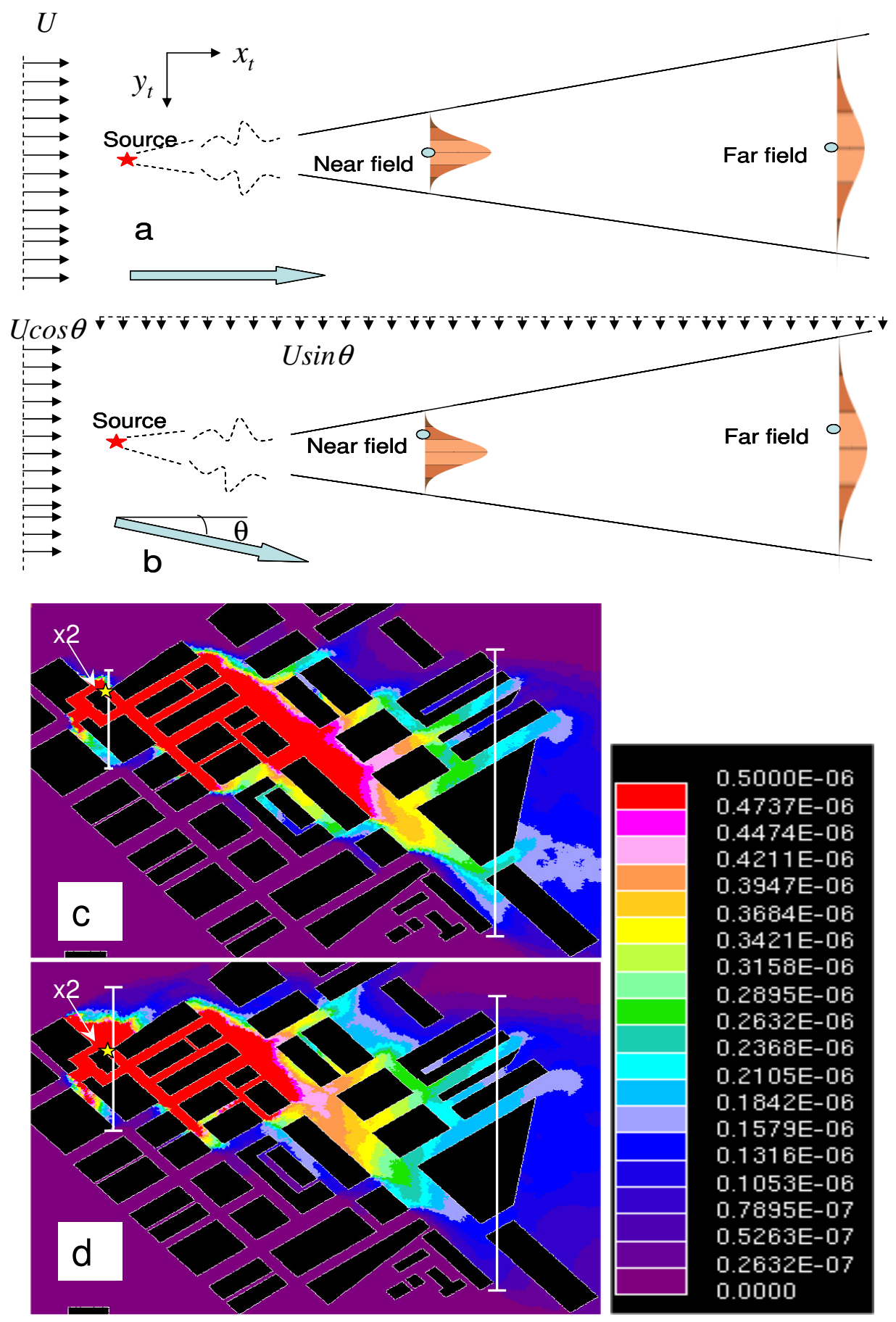

Fig. 12 Effects of long-time wind direction variations on dispersion. A conceptual sketch of a plume (a) in a wind in $x_{t}$ direction at time $t$ and (b) in a wind with direction changed to $\theta$ bearing clockwise to $x_{t}$ direction at time $t+\delta t$. A comparison of 30-min averaged concentration contour at $z / h_{m}=0.9$ (c) using 30-min averaged BT Tower data and (d) using 30-sec averaged BT Tower data (Fig. 6). 
far field, e.g. in Regent's Park, is hardly discerned. All these results seem consistent with the speculation above.

It is often suggested that the plume depth in the vertical direction in full scale urban environments is much greater than that in wind-tunnel experiments in which steady and neutral wind conditions are imposed. Thermal convection likely plays a non-negligible role in this aspect. Field experiments (Wood et al., 2009, 2010) show that the Monin-Obukhov length can be frequently less than $-19 \mathrm{~m}$ in London in daytime June. This suggests that further numerical and wind-tunnel modelling are needed to include the thermal convection. It may not be very surprising that the variation of the wind direction significantly increases the plume width in the horizontal direction, in particular in the near field. It is also of interest to know whether it affects the plume depth in the vertical direction. Vertical profiles of the mean concentration at the major intersection in varying and steady winds were studied. The variation of wind direction did increase the plume depth in the vertical direction, but not as significantly as in the horizontal direction. This might be reasonable for large-eddy simulations in neutrally stratified winds. It is to be noted that the domain height $L_{z}$ was approximately $9 h_{m}$. The vertical depth $\sigma$ of a plume is defined as the height where the mean concentration is half of the maximum mean at a station. At the major intersection, $L_{z} / \sigma$ is approximately 5. This may suggest that the domain height is probably enough to prevent serious constraint of vertical plume spread in neutral conditions. In order to model the effect of the thermal convection on dispersion, computations with a greater domain height might be necessary, in which a greater plume width is expected.

\section{Final Discussion and Conclusions}

In order to investigate the effect of wind direction on dispersion, a comparison of large-eddy simulations of point-source dispersion over the DAPPLE site in $-51.4^{\circ}$ (i.e. oblique) and $-90^{\circ}$ (i.e. perpendicular to the windward faces of the buildings) winds was performed. Both the velocity and scalar fields predicted by the numerical simulations were validated using wind-tunnel measurements. One conclusion can be drawn here - the statistics and the instantaneous concentration of the released tracer are sensitive to the wind direction in urban environments. Also we noticed that the local arrangement of the buildings at the DAPPLE site produced significant effects on the direction of the spatially-averaged time-mean velocity within the canopy. This raises the issue of how to parametrize the data within the urban canopy (obtained from street-scale models or field experiments) to be used in a mesoscale meteorological model with the first grid point below the urban canopy height.

Our long-term objective is to use the mesoscale wind conditions from the mesoscale meteorological models with a resolution in the order of $1 \mathrm{~km}$ to drive the street-scale large-eddy simulations with resolution down to $1 \mathrm{~m}$. However we have not yet found an appropriate computational approach to bridge the large gap between the smallest time scale of the mesoscale meteorological models (e.g. 1 hour) and the turbulence integral scale (e.g. 1 minute). Instead we turned to using realistic wind conditions measured at the $190 \mathrm{~m}$ height on BT Tower as boundary conditions to drive the street-scale large-eddy simulations of flows and dispersion over the DAPPLE site, in which the turbulent motions within the integral length scales at the inlets were generated by our recently developed efficient inflow approach. The predicted dispersion was in significantly better agreement with the field measurements than when steady wind conditions were used, in particular in the near-source field. It has been shown that the dispersion in the near-source field is affected more than that in the far field by long-time wind-direction variations. It was also noticed that the plume width in the varying wind conditions is greater than that in the steady wind conditions not only in the horizontal direction but also in the vertical direction. 
This validation helps to determine how to use current mesoscale meteorological data (e.g. the UM or WRF models) to drive micro- (street) scale computations. But there are some issues left to be addressed. In the current study, a neutral thermal stability was assumed. However, it has been found that the urban environments of a large European city, e.g. London, are always in weakly unstable conditions (Wood et al., 2010). The effects of thermal convection may thus also need to be considered in the computations.

Acknowledgements The research is partially supported by NERC under the Urban Meteorology theme of the Universities Weather Research Network (UWERN, Grant No. R8/H12/38). The author is grateful to Prof I.P. Castro for critical comments and some of the data analysis. The author is very grateful to Drs V.B.L. Boppana, P. Hayden and C. Wood for providing technical support, wind tunnel and field data. The author is also grateful to the three anonymous referees for their useful comments. All of the computations are done using CD-adapco's Star-CD V4. The computations were performed on the Iridis computational system, University of Southampton, and HECToR at the UK's supercomputer centre.

\section{References}

Arnold S, ApSimon H, Barlow J, Belcher S, Bell M, Boddy D, Britter R, Cheng H, Clark R, Colvile R, Dimitroulopoulou S, Dobre A, Greally B, Kaur S, Knights A, Lawton T, Makepeace A, Martin D, Neophytou M, Neville S, Nieuwenhuijsen M, Nickless G, Price C, Robins A, Shallcross D, Simmonds P, Smalley R, Tate J, Tomlin T, Wang H, Walsh P (2004) Introduction to the DAPPLE air pollution project. Sci Total Environ 332:139-153

Balogun AA, Tomlin AS, Wood CR, Barlow JF, Belcher SE, Smalley RJ, Lingard JJN, Arnold SJ, Dobre A, Robins AG, Martin D, Shallcross DE (2010) In-street wind direction variability in the vicinity of a busy intersection in central london. Boundary-Layer Meteorol 136:489-513

Belcher SE (2005) Mixing and transport in urban areas. Phil Trans Roy Soc 363:2947-2968

Branford S, Coceal O, Thomas TG, Belcher SE (2011) Dispersion of a point-source release of a passive scalar through an urban-like array for different wind directions. Boundary-Layer Meteorol 139:367-394

Britter RE, Hanna SR (2003) Flow and dispersion in urban areas. Ann Rev Fluid Mech 35:469-496

Carpentieri M, Robins AG, Baldi S (2009) Three-dimensional mapping of air flow at an urban canyon intersection. Boundary-Layer Meteorol 133:277-296

Castro IP, Cheng H, Reynolds R (2006) Turbulence over urban-type roughness:decutions from wind-tunnel measurements. Boundary-Layer Meteorol 118:109-131

Cheng H, Robins AG (2004) Wind tunnel simulation of field tracer release in London. In: Zhuang FG, Li JC (eds) Recent Advances in Fluid Mechanics, Tsinghua University Press \& Springer-Verlag, pp 801-804

Claus JM, Castro IP, Xie ZT (2009) Wind direction effects on urban like roughness: an LES study. In: Eckhardt B (ed) Advances in Turbulence XII, Proceedings of the 12th EUROMECH European Turbulence Conference, vol 132, Springer, pp 409-412

Coceal O, Thomas TG, Castro IP, Belcher SE (2006) Mean flow and turbulence statistics over groups of urban-like cubical obstacles. Boundary-Layer Meteorol 121:491-519

Davidson MJ, Mylne KR, Jones CD, Phillips JC, Perkins RJ, Fung JCH, , Hunt JCR (1995) Plume dispersion through large groups of obstacles - a field investigation. Atmos Environ 29:3245-56

Davidson MJ, Snyder WH, Lawson RE, , Hunt JCR (1996) Wind tunnel simulations of plume dispersion through groups of obstacles. Atmos Environ 30:3715-31

Fernando HJS, Zajic D, di Sabatino S, Dimitrova R, Hedquist B, Dallman A (2010) Flow, turbulence, and pollutant dispersion in urban atmospheres. Phys Fluids 22:051,301-1-20

Hanna SR, Tehranian S, Carissimo B, Macdonald RW, Lohner R (2002) Comparisons of model simulations with observations of mean flow and turbulence within simple obstacle arrays. Atmos Environ 36:50675079 
Hellstena A, Rautaheimo P (1999) In: Hellstena A, Rautaheimo P (eds) Proceedings, 8th ERCOFTAC / IAHR / COST Workshop on Refined Flow Modelling, June 17-18. Helsinki Univ. Tech. Finland., 207 pp

Kanda M, Moriwaki R, Kasamatsu F (2004) Large-eddy simulation of turbulent organized structures within and above explicitly resolved cube arrays. Boundary-Layer Meteorol 112:343-368

Lim HC, Castro IP, Hoxey RP (2007) Bluff bodies in turbulent boundary layers: Reynolds-number issues. J Fluid Mech 571:97-118

MacDonald RW, Griffiths RF, Hall DJ (1998) A comparison of results from scaled field and wind tunnel modelling of dispersion in arrays of obstacles. Atmos Environ 32:3845-3862

Martilli A (2002) Numerical study of urban impact on boundary layer structure: sensitivity to wind speed, urban morphology, and rural soil moisture. J Appl Meteor 41:1247-1266

Mochida A, Iizuka S, Tominaga Y, Lun YFI (2010) Up-scaling CWE models to include mesoscale meteorological influences. In: The 5th International Symposium on Computational Wind Engineering, Chapel Hill, North Carolina, USA. ftp://ftp.atdd.noaa.gov/pub/cwe2010/Files/Papers/581_Mochida.pdf

Moeng CH, Dudhia J, Klemp J, Sullivan P (2007) Examining two-way grid nesting for large eddy simulation of the PBL using the WRF model. Mon Weather Rev 135:2295-2311

Niceno B, Hanjalić K (2002) Turbulent heat transfer from a multi-layered wall-mounted cube matrix: A large eddy simulation. Int J Heat Fluid Flow 23:173-185

Peric M (2004) Flow simulation using control volumes of arbitrary polyhedral shape. ERCOFTAC Bulletin 62:25-29

Santiago JL, Dejoan A, Martilli A, Martin F, Pinelli A (2010) Comparison between large-eddy simulation and reynolds-averaged navier-stokes computations for the must field experiment. part i: Study of the flow for an incident wind directed perpendicularly to the front array of containers. Boundary-Layer Meteorol 135:109-132

Solazzo E, Sabatino SD, Aquilina N, Dudek A, Britter R (2010) Coupling mesoscale modelling with a simple urban model: The lisbon case study. Boundary-Layer Meteorol 137:441-457

STAR-CD (2009) Cd-adapco's ccm user guide. STAR-CD Version 408

Stoesser T, Mathey F, Frohlich J, Rodi W (2003) LES of flow over multiple cubes. ERCOFTAC Bulletin 56:15-19

Tseng YH, Meneveau C, Parlange MB (2006) Modeling flow around bluff bodies and urban dispersion using large eddy simulation. Environ Sci Technol 40:2653-2662

Wood CR, Barlow JF, Belcher SE, Dobre A, Arnold SJ, Balogun AA, Lingard JJN, Smalley RJ, Tate JE, Tomlin AS, Britter RE, Cheng H, Martin D, Petersson FK, Shallcross DE, White IR, Neophytou MK, Robins AG (2009) Dispersion experiments in central London: the 2007 DAPPLE project. Bull Amer Meteor Soc 90:955-969

Wood CR, Lacser A, Barlow JF, Padhra A, Belcher SE, Nemitz E, Helfter C, Famulari D, Grimmond CSB (2010) Turbulent flow at 190 metres above London during 2006-2008: a climatology and the applicability of similarity theory. Boundary-Layer Meterol 137:77-96

Xie ZT, Castro IP (2006) LES and RANS for turbulent flow over arrays of wall-mounted cubes. Flow Turbul and Combust 76:291-312

Xie ZT, Castro IP (2008) Efficient generation of inflow conditions for large-eddy simulation of street-scale flows. Flow Turbul and Combust 81:449-470

Xie ZT, Castro IP (2009) Large-eddy simulation for flow and dispersion in urban streets. Atmos Environ 43:2174-2185

Xie ZT, Castro IP (2010) Coupled modelling of flow and dispersion in urban environments. In: The 5th International Symposium on Computational Wind Engineering, Chapel Hill, North Carolina, USA. ftp://ftp.atdd.noaa.gov/pub/cwe2010/Files/Papers/558_Castro.pdf 
Xie ZT, Coceal O, Castro IP (2008) Large-eddy simulation of flows over random urban-like obstacles. Boundary-Layer Meteorol 129:1-23

Yamada T (2010) Downscaling Mesoscale Meteorological Models for Computational Wind Engineering Applications. In: The 5th International Symposium on Computational Wind Engineering, Chapel Hill, North Carolina, USA. ftp://ftp.atdd.noaa.gov/pub/cwe2010/Files/Papers/341_yamada.pdf

Yoshizawa A (1986) Statistical theory for compressible turbulent shear ows, with the application to subgrid scale modelling. Phys Fluids 29:2152-2164 